\title{
Frequency Discrimination in the Sense of Flutter: Psychophysical Measurements Correlated with Postcentral Events in Behaving Monkeys
}

\author{
V. B. Mountcastle, M. A. Steinmetz, and R. Romo ${ }^{a}$ \\ The Philip Bard Laboratories of Neurophysiology, Department of Neuroscience, The Johns Hopkins University School of \\ Medicine, Baltimore, Maryland 21205
}

\begin{abstract}
The capacities of humans and monkeys to discriminate between the frequencies of mechanical sinusoids delivered to the glabrous skin of the hand have been measured in psychophysical experiments. The 2 primates have similar capacities; they make discriminations with Weber fractions that change little over the frequency range from 20 to $200 \mathrm{~Hz}$. The discriminatory capacities are similar whether stimuli are received passively or acquired actively. Combined experiments have been made in monkeys in which the electrical signs of the activity of quickly adapting (QA) and slowly adapting (SA) neurons of postcentral areas $3 \mathrm{~b}$ and 1 were recorded, both in the working state as the animal made discriminations and in the irrelevant state in which the stimuli did not guide behavior. The neuronal responses were analyzed in terms of discharge rates, periodicities in the neuronal discharges, and harmonic contents. It was shown that discriminatory capacity depends upon the period lengths in the sets of periodically entrained activity evoked by stimuli readily discriminated, and not upon the small differences in rates of discharge evoked by those stimuli. The periodicities were shown by harmonic analysis to be sharply limited to stimulus frequencies. Low-frequency stimuli evoke periodicities at the second and third harmonics in some neurons, in addition to strongly periodic signals at the fundamental frequency of the stimuli. Their presence does not appear to interfere with frequency discrimination. Neuronal responses recorded in the stimulus-irrelevant state were not distinguishable from those recorded as monkeys made discriminations. The responses of SA neurons, recorded under similar conditions, resembled those of QA neurons in almost every feature, but reasons are given for concluding that the SA system plays no role in frequency discrimination in the sense of flutter.
\end{abstract}

\footnotetext{
Received Feb. 14, 1990; revised Apr. 30, 1990; accepted May 7, 1990.

We acknowledge with appreciation the partial support of this research from U.S. Public Health Service Grants 5 P01-NS-20868 and 1 R01 NS-27776.

Correspondence should be addressed to Vernon B. Mountcastle, M.D., Philip Bard Laboratories of Neurophysiology, Department of Neuroscience, The Johns Hopkins University School of Medicine, 725 North Wolfe Street, Baltimore, MD 21205 .

Present address: Departamento de Neurosciencias, Instituto de Fisiologia Celular, Universidad Nacional Autonoma de Mexico, Apartado Postal 70-600, Mexico, D.F., Mexico.

Copyright (C) 1990 Society for Neuroscience $0270-6474 / 90 / 093032-13 \$ 03.00 / 0$
}

A major problem in understanding the cortical neural mechanisms in perception is to determine whether the temporal order in which impulscs are discharged by central neurons, driven by sensory stimuli, carries significant information about stimulus parameters; in particular, whether differences in the temporal patterns of activity evoked in the same sets of cortical neurons by different stimuli may serve as neural discriminanda adequate for identifying and differentiating between those stimuli. The question of temporal-order coding has long been studied in experimental neurophysiology (for reviews, see Moore et al., 1966; Perkel et al., 1967; Perkel, 1970; Klemm and Sherry, 1981, 1982; Gerstein, 1987), but only occasionally has it been possible to show that serial-order codes are essential guides for sensory behavior.

The experiments described here were designed to address this problem directly. We chose to study frequency discrimination in the sense of flutter, a form of cutaneous sensibility we have studied in several earlier investigations (Talbot et al., 1968; Mountcastle et al., 1969, 1972; LaMottc and Mountcastle, 1975, 1979). It offers several advantages for our purpose. First, a great deal is known about the sensory capacities of primates in the sense of flutter and of the peripheral transduction and encoding of the relevant stimuli, which are mechanical sinusoids of 5-40 $\mathrm{Hz}$. Second, it is a form of somatic sensation readily adapted for study in the combined behavioral-neurophysiological experiments we wished to execute. Finally, there is good evidence that mechanical sinusoids of slightly different frequencies, between which primates can discriminate, evoke different temporal patterns of activity in the same sets of primary afferent nerve fibers, in the same linking neurons of the somatic afferent system, and in the same sets of neurons in the postcentral somatic sensory cortex.

In an earlier set of experiments, we examined the capacity of human subjects to make frequency discriminations between flutter stimuli and analyzed the results in the context of decision theory (Mountcastle et al., 1969). A parallel series of experiments employing single stimuli in the same frequency ranges was made in unanesthetized, neuromuscularly blocked (denervated-head) monkeys. It was found that the variance of the presumed sensory events from the first analysis changed pari passu with the variances of the periodicities in the responses of postcentral neurons evoked by similar stimuli delivered to the monkeys' hands. This covariance led to the hypothesis that it is the periodicities in the entrained postcentral activity that adequately represent the frequencies of peripheral stimuli, that the periodicity depends upon the serial order of impulse inter- 
vals, and that differences in the lengths of the periods in sets of evoked activity might serve as the neural discriminanda for frequency in the sense of flutter.

It was our aim in the experiments presented here to test this hypothesis directly by recording the activity of postcentral neurons evoked by mechanical sinusoids delivered to the glabrous skin of the hands of monkeys as they worked in frequencydiscrimination tasks. We have also recorded this evoked activity under exactly similar conditions, except that the alert monkeys worked at no task. This allowed us to compare the postcentral neural responses when the stimuli were relevant to when they were irrelevant as guides for sensory bchavior.

We first examined the capacities of human and monkey subjects to discriminate between mechanical sinusoids of different frequencies and confirmed again that the capacities of the 2 primates are virtually identical (LaMotte and Mountcastle, 1975).

Our results support the idea that serial-order coding is critical for the postcentral representation of the frequency of flutter stimuli, and that the periodicities created by that order, revealed by harmonic analysis, compose the critical neural discriminanda at the postcentral level for frequency discrimination. We found no differences in postcentral neural responses between those evoked by relevant and those evoked by irrelevant stimuli. This indicates that the modification of sensory performance produced by shifts of attention, or in other control states, must be exerted at some "more central" level than the postcentral gyrus in the processing networks leading from there to perception and to differential motor response.

\section{Materials and Methods}

Animal training and primate psychophysics. Human subjects (3) were members of our laboratory staff. Monkey subjects (4) lived in $4 \mathrm{ft}^{3}$ cages in a room housing 12-15 monkeys. All were tuberculin and $\mathrm{B}$-virus negative. They sat in restraining chairs for daily 3 -hr training/testing sessions, then were returned to their living cages. Monkeys were deprived of water during training and testing periods; each earned or was given $30-35 \mathrm{ml}$ water $/ \mathrm{kg}$ body weight each day and allowed free access to water 1 day each week

The 3 humans and 2 monkeys were trained and tested in the passive mode of the frequency-discrimination task (Fig. 1). Humans sat facing a hcavy mctal tablc with left arms restrained in a half-cast and handfinger mold. Monkeys in restraining chairs were placed in a slot in the same table (for them, air suspended), each with an arm and hand similarly placed. Stimuli were delivered to the distal pads of fingers 2,3 , or 4 with a Chubbuck linear motor stimulator bearing a rounded Lucite probe with a tip diameter of 1 or $2 \mathrm{~mm}$. Subjects initiated a trial when they detected a step indentation of the skin $(0.5 \mathrm{~mm})$ by interrupting a light beam with the right hand. They were required to maintain that interruption through a variable prestimulus delay $(0.5-4.0 \mathrm{sec})$ until 2 stimuli, each of $1-\mathrm{sec}$ duration, had been delivered in sequence, with interstimulus intervals varying between 0.5 and $2.0 \mathrm{sec}$. They then were to indicate detection of the end of the second stimulus by removing the right hand from the light path within a $0.2-0.6 \mathrm{sec}$ reaction-time window and project the right hand to 1 of 2 target switches to indicate whether the frequency of the second stimulus was higher or lower than that of the first. Although the stimulator was noiseless, a broad-band masking noise was delivered via a speaker behind the subject's head. Monkeys were rewarded with a drop of liquid for each correct discrimination; a soft click indicated correct choice to human subjects. Detection, discrimination, or inter/intratrial errors were signaled by brief increases in the volume of the masking noise.

Two human subjects were trained and tested in the active form of the discrimination task outlined in Figure 1. These subjects initiated each trial upon the appearance of a visual "go" signal by applying the distal pad of the left third finger to the center of a flat, circular manipulandum $6 \mathrm{~cm}$ in diameter. They were required to maintain upon the manipulandum a force within a preset range of 70-140 gm while awaiting the 2 successive mechanical oscillations of the manipulandum. The programmed force windows and the force exerted by the subjects were indicated visually with a LED bar display. The subject maintained force within the window throughout the test sequence shown in Figure 1, indicated detection of the end of the second stimulus by releasing force, and his choice of higher or lower frequency of the second stimulus by projecting his hand to 1 of the 2 target switches. Masking sounds and an audible click indicating correct choice were delivered as described above.

Monkey training. Monkeys were first traincd to detect the end of a single stimulus of $30-\mathrm{Hz}$ frequency and $1-\mathrm{sec}$ duration. The durations of the second stimuli were first lengthened and then interrupted in midcourse. The interruption was then itself lengthened and varied in duration, and training continued until the animal was detecting the ends of the second stimuli, with varying pre- and interstimulus periods. The frequencies of the second stimuli were then changed, for example, to $50 \mathrm{~Hz}$ for one class of trials and $10 \mathrm{~Hz}$ for another, with a base frequency of $30 \mathrm{~Hz}$. These 2 classes were then delivered in a pseudorandom order on successive trials, and the correct panel switch was lighted to guide the choice of response. However, we found that an effective method of training was to sound a $1000-\mathrm{Hz}$ tone simultaneously with a second stimulus of $50 \mathrm{~Hz}$, and a $100-\mathrm{Hz}$ tone with a second stimulus of $10 \mathrm{~Hz}$. Training then progressed rapidly without lighting the panel switches, and within 2-4 d, we were able to eliminate the cuing sounds. The monkeys were then introduced to full but wide discrimination sets. These sets were gradually narrowed to $2-$, then $1-\mathrm{Hz}$ frequency differences between the first and second stimuli, in different classes. Full training to minimal difference limens required about 4 months, after which time psychophysical testing began.

Construction of stimulus-control sets for frequency discrimination. The subjective intensity of a mechanical sinusoid delivered to the skin changes with stimulus frequency (Goff, 1967; LaMotte and Mountcastle, 1975). Therefore, it is necessary for studies of frequency discrimination to adjust the amplitudes of the comparison stimuli of different frequencies to match in subjective intensity that of the base stimuli with which frequency discrimination is to be attempted. We constructed equal subjective magnitude functions in the following way: Experienced human subjects received stimuli in the passive mode of the task of Figure 1, in stimulus sets in which the base stimuli of constant frequency and amplitude were followed by the second stimuli of a different constant frequency but with amplitudes that varied randomly from trial to trial above and below that of the base stimuli. Subjects were asked to ignore pitch differences and to indicate whether the second stimuli were greater or lesser in subjective intensity than the first. The results were plotted as psychophysical functions, and the equal subjective magnitudes were calculated from them. Repetition of this experiment at the several frequencies making up a frequency-discrimination control set allowed construction of sets with appropriate amplitude values for the comparison stimuli. This method yielded results comparable with those of LaMotte and Mountcastle (1975).

We have made 2 control experiments to test whether our subjects were attending to and discriminating between the frequency and not the intensity of the stimuli they received. First, both human and monkey subjects trained in frequency discrimination suffered no deterioration in performance when the amplitudes of the comparison stimuli were surreptitiously changed on randomly chosen trials. Second, we constructed special stimulus sets in which we combined 2 sets with different base stimuli; for example, one set in which the base stimulus of 30 was followed by comparison stimuli ranging from 22 to $38 \mathrm{~Hz}$ in $2-\mathrm{Hz}$ steps, and a second in which the base stimulus of $40 \mathrm{~Hz}$ was followed by comparison stimuli ranging from 32 to $48 \mathrm{~Hz}$ in $2-\mathrm{Hz}$ steps. Trials in this combined set were sequenced randomly. Under these circumstances, frequencies from 32 to $38 \mathrm{~Hz}$ must be correctly identified as "higher" on some trials and "lower" on others. Our human subjects were able to make the 2 sets of discriminations simultaneously with little deterioration of performance. For example, 1 human subjcct attained a net correct score of $84 \%$ when making discriminations in the zone of overlap of the 2 sets when they were delivered separately and a net correct score of $74 \%$ when trials from the 2 sets were sequenced randomly; his performance in the latter was $88 \%$ of that in the former.

The combined behavioral-neurophysiological experiment. We adapted to the waking monkey the Reitboeck method of multiple-microelectrode recording (Reitboeck, 1983a, b). The system allows independent movement of any 1 of 7 microelectrodes in $2-\mu \mathrm{m}$ steps. Electrodes were carried in stainless steel guide tubes that converged into a $7-\mathrm{mm}$ stainless steel implant thimble, and all tubes opened flush with its distal surface. For 
Figure 1. Schematic outlines of active and passive modes of frequency-discrimination task. Descriptions of the task sequences, stimulus-set construction, and psychophysical experiment execution are given in the text.

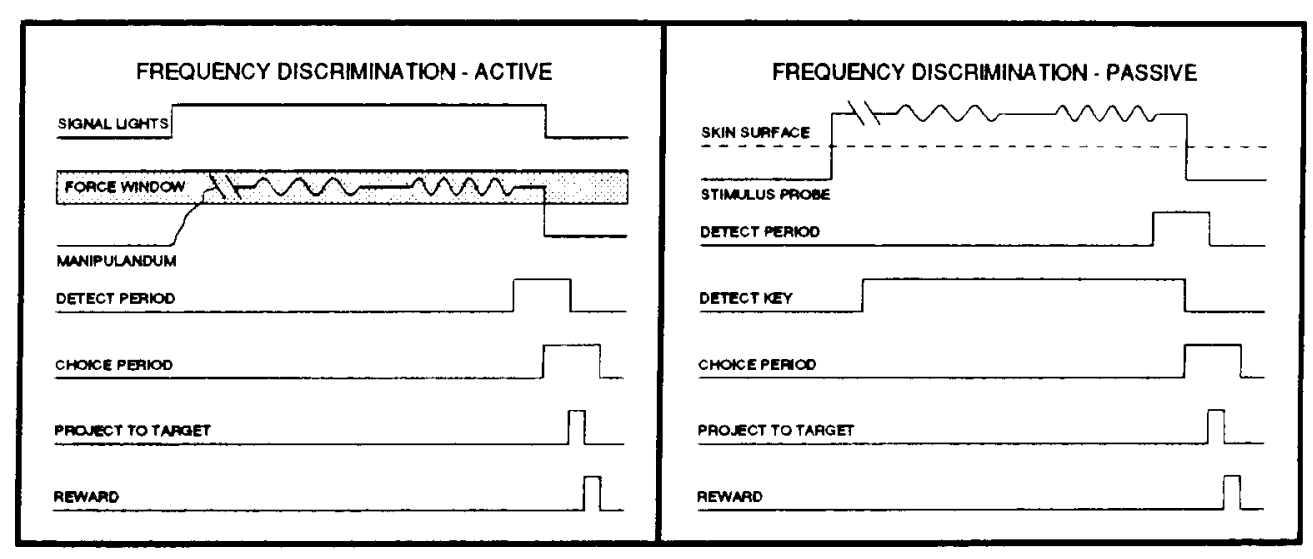

recording, the implant thimble was covered with a rubber dam and sealed with an o ring into a previously drilled hole in the acrylic cap and skull, distal surface flush with the dura. Electrodes were passed individually through the rubber dam and dura and into the cortex. We used linear arrays of electrodes spaced $600 \mu \mathrm{m}$ apart, in lines that could be centered or placed 1 or $2 \mathrm{~mm}$ eccentrically in different implant thimbles and rotated in the $x-y$ plane to access any chosen position. The $7-\mathrm{mm}$ hole through acrylic and bone was drilled the day before recording, under ketamine anesthesia, and closed with a plug. On each recording day, the plug was removed to allow insertion of the implant thimble. A local antibiotic solution (gentamicin sulfate, $2 \mathrm{mg} / \mathrm{ml}$ ) was used in the small opening to prevent infection. The Reitboeck microelectrodes were quartz/glass filaments with an outside diameter of $80 \mu \mathrm{m}$ and $30-\mu \mathrm{m}$ central cores filled with a tungsten-platinum alloy (Reitboeck, $1983 \mathrm{a}, \mathrm{b})$. The filaments were drawn in a helium-filled chamber with a restrictive heating element, then ground to a conical tip on the diamond wheel. We have found satisfactory those filaments that taper gently to a $10-12-\mu \mathrm{m}$ diameter, then more abruptly to a point of exposed metal; they have impedances of 2-4 M $\Omega$ at $1000 \mathrm{~Hz}$.

The multichannel recording system. The system allowed simultaneous data collection from 8 channels and independent $z$-axis position of each of the 7 electrodes. Each channel included impedance transforming and testing devices and an equalizing amplifier with band-pass filters and a differential-amplitude discriminator. The output of each discriminator was directed to a selected bit of the input register of a custom-built computer interface. Four dual-beamed oscilloscopes were used for display, 2 channels per scope. The system was controlled with a Motorola 6809 microprocessor interfaced to a standard terminal. Commands from the terminal would (1) connect signals from any 1 of the 7 electrodes to any 1 of the 8 discriminators, (2) provide a measurement of electrode impedance, (3) control the movement of the microelectrodes, and (4) register the $z$-axis position of each electrode.

Experimental control and data collection and analysis were accomplished by a system of PDP 11-84 computers and Microvax work stations linked with Ethernet hardware and DecNet software. Real-time stimulus and behavioral control and data collection were carried out on 1 PDP 11-84 using standard computer interfaces. The intervals between neural events and behavioral events on each processing channel were collected and stored with a resolution of $100 \mu$ sec. On-line raster displays were generated on VT330 graphic terminals allowing continuous display of neural activity from each of the 8 processing channels. Upon completion, data files were copied to the Microvax for off-line analysis.

Data analysis. Our initial survey of neural data included examination of raster plots and of PST and cycle histograms synchronized on stimulus events. These analyses provided confirmation of the functional properties of each cortical neuron studied. Cortical neurons were classified as slowly adapting, quickly adapting, or Pacinian on criteria given below. Cyclic entrainment of neuronal responses was evaluated by plotting expectation density (i.e., autocorrelation) functions from impulse-interval train data, as described by Poggio and Viernstein (1964). The dependence of the observed rhythmicity on the sequential order of impulse intervals, rather than on the dominant intervals, was assessed by plotting renewal density functions computed by repeating the expectation density analysis after a random shuffling of the sequential order of impulse intervals.
Fourier analysis was used to quantify the strength and periodicity of the rhythmic entrainment of cortical neuronal responses (Bloomfield, 1976; Bracewell, 1986). Unsmoothed frequency histograms were constructed from the neural discharges recorded during the 1-sec stimulus periods using 1 -msec bins averaged across repeated trials. Fast Fourier transforms of the frequency counts provided the periodogram ordinate values from $1 \mathrm{~Hz}$ through $500 \mathrm{~Hz}$ in $1-\mathrm{Hz}$ steps. The spectral density (power fraction) at individual frequencies was expressed as the ratio of the periodogram ordinate value at that frequency to the sum of all the ordinate values. Statistical significance of individual power fractions was tested using Shimshoni's rank-order modifications of Fisher's significance test for harmonic analysis (Fisher, 1929; Shimshoni, 1971).

Psychometric functions were obtained for the human and monkey discriminations between vibratory stimuli of different frequency by plotting the percent of comparison stimuli identified as higher in frequency than the base stimuli as a function of the frequency of the comparison stimulus. Logistic functions of the form: $f(x)=100 /\left[1+e^{(x+b)}\right]$ were fitted to the data using an iterative method for a least squares solution (Draper and Smith, 1981). All regressions were shown to be significant by analysis of variance $(p<0.05)$. Difference limens (DLs) were computed as $1 / 2$ the difference between the stimulus frequency identified as higher than the standard on $75 \%$ of the trials and that frequency identified as higher on $25 \%$ of the trials. These values were read directly from the fitted functions, expressed in terms of cycle lengths in msec, and Weber fractions were calculated from them.

Anatomical studies. After the experiments, 3 of the 4 monkeys of Table 1 (109 is still alive) were anesthetized with ketamine $(6 \mathrm{mg} / \mathrm{kg}$ ) and intravenous sodium pentobarbital $(25 \mathrm{mg} / \mathrm{kg})$ and perfused throughout the right ventricle with $0.9 \% \mathrm{NaCl}$ followed by $4 \%$ formalin. The brain was removed and suspended in formalin. Later, a block from each hemisphere containing the pre- and postcentral gyri was embedded in celloidin and sectioned at $20 \mu \mathrm{m}$ along a line as normal as possible to the central sulcus. Every section was mounted and stained with $0.5 \%$ thionin. Search of these sections with both low- and high-power light microscopy has revealed no sign of the 178 microelectrode penetrations known to have entered the postcentral gyri in these brains in the daily acute experiments. We attribute this to the atraumatic nature of the Reitboeck electrode, with its smooth shaft of constant diameter $(80 \mu \mathrm{m})$, and to the 6-hr recording period, which must limit glial reaction. In contrast, the tracks of the electrodes implanted chronically and left until death in the 2 hemispheres of monkey 105 are sharply outlined by glial cells and easily reconstructed. They are perfectly straight and provide evidence that the Reitboeck electrode does not wander during penetration of brain tissue. These tracks, as well as those of the implanted guide tubes, allowed us to determine that the electrodes inserted into the hemispheres of these monkeys passed into area 1 and, when carried deeply enough, into area $3 \mathrm{~b}$. The functional properties of the cells described below lend strength to our conclusions; therefore, we state that the neurons entered in the data base of Table 1 were all located in areas 1 and $3 b$ of the postcentral gyrus.

\section{Results}

\section{Data base}

The psychophysical results were obtained in experiments in 3 human and 2 monkey subjects. The humans had a long expe- 
rience in this type of experiment in somesthesis. Each monkey $(108,109)$ was trained for about 4 months, in the manner described above, before testing began.

The neurophysiological results were obtained from 4 monkeys. They were carried out using several different protocols for study of different aspects of somesthesis (detection, discrimination, etc.). We abstracted from the data base of Table 1, for the present purpose, neurons studied with frequency-discrimination control sets: 30 neurons studied as monkey 109 performed the passive discrimination task of Figure 1, and 46 neurons as alert animals $(105,106,107)$ performed no task; for the latter, the stimuli were irrelevant and did not control behavior.

\section{Neuron identification}

Neurons entered the data base of Table 1 if (1) they were assumed on criteria given below to be located in areas 1 or $3 \mathrm{~b}$, (2) their receptive fields were located on the digital glabrous skin, and (3) they responded to gentle mechanical stimulation of the glabrous skin of the contralateral hand. It is generally accepted that the neurons of areas $3 \mathrm{~b}$ and 1 can be described and classified in terms of the functional properties of the sets of large mechanoreceptive afferent fibers that innervate the skin of the hand (Mountcastle et al., 1969), a specificity undoubtedly produced by a restricted cross-modal projection and maintained in part by dynamic mechanisms. There are 4 such sets: the quickly adapting afferents (QA) terminating in Meissner corpuscles, the slowly adapting class I (SA-I) terminating in the Merkel's discs, the slowly adapting class II (SA-II) terminating in Ruffini organs, and the Pacinian (PC) afferents terminating in Pacinian corpuscles. Cortical PC neurons are readily identified by their high-pass property, for they respond to low-amplitude, high-frequency mechanical sinusoids $(60-400 \mathrm{~Hz})$ delivered to the skin and not to low frequencies $(5-40)$; their receptive fields are large and poorly defined. SA-I and QA cortical neurons have comparable band passes in the low-frequency range of flutter $(5-40 \mathrm{~Hz}$ ). They cannot be differentiated by receptive-field properties but are readily differentiated by their differences in rate of adaptation to steady skin indentations. We have found no neurons of areas $3 b$ and 1 of the monkey postcentral gyrus with properties similar to those of the SA-II afferents. These were identified in studies of the first-order fibers innervating the glabrous skin of the human hand. However, direct electrical stimulation of identified SA-II fibers in human peripheral nerves evokes no sensation (Torebjork and Ochoa, 1980; Ochoa and Torebjork, 1983; Torebjork et al., 1984, 1987; Vallbo and Johannson, 1984; Vallbo et al., 1984).

\section{Neuron location}

Neurons were assumed to be located in area 2 if they subtended large, multifingered receptivc ficlds and if such ficlds were observed from cell to cell in cortical depth during a penetration, a characteristic emphasized by Darian-Smith et al. (1984) and Iwamura et al. (1980); none were included in the present study. We found the presumed transition from area 2 to area 1 to be sharp, based on the change from multifingered to single-fingered receptive fields described by those authors. Equally sharp was the presumed transition from area 1 to area 3 , based on the reversal in the progression and regression of the receptive fields from tip to base and from base to tip of the fingers. This transition has been correlated with the cytoarchitectural transition from area 1 to 3 b by Kaas et al. (1979) and Darian-Smith et al.
Table 1. Data base for the series of experiments from which the observations described in this paper were drawn

\begin{tabular}{lrrrrrrrr} 
Hemi- & & \multicolumn{8}{c}{ Neurons studied } & & \\
\cline { 5 - 8 } spheres & Sets & Pens & QA & SA & PC & SP & Total & Runs \\
\hline $105-\mathrm{L}$ & 12 & 60 & 52 & 12 & 1 & 2 & 67 & 369 \\
$105-\mathrm{L}$, CR & 1 & 5 & 6 & - & 3 & - & 9 & 94 \\
$105-\mathrm{R}$ & 10 & 53 & 63 & 5 & 18 & 9 & 95 & 415 \\
$105-\mathrm{R}, \mathrm{CR}$ & 1 & 4 & 4 & 1 & - & 2 & 7 & 57 \\
$106-\mathrm{R}$ & 7 & 24 & 29 & 1 & 2 & - & 32 & 129 \\
$107-\mathrm{R}$ & 12 & 41 & 44 & 26 & - & 1 & 71 & 350 \\
$109-\mathrm{R}$ & 9 & 13 & 58 & 2 & 4 & 2 & 66 & 225 \\
Sums & 52 & 200 & 256 & 47 & 28 & 16 & 347 & 1639
\end{tabular}

Monkey 108 was used only in psychophysical experiments and is therefore not included above. CR indicates instances of chronic implantation of microelectrodes. Sets is the number of cortical insertions of the 7-electrode array. Pens indicates the number of successful microelectrode penetrations into areas $3 \mathrm{~b}$ and 1 of the postcentral gyrus. SP indicates neurons with special properties. Runs are the number of computer-controlled stimulus sets run.

(1984). All neurons activated from single-fingered receptive fields and located more than $2000 \mu \mathrm{m}$ below the top of neural activity in a microelectrode penetration were classed in area $3 \mathrm{~b}$. We found both SA and QA neurons commonly occur in both areas $3 \mathrm{~b}$ and 1 , based on these criteria. Our sample of PC neurons is small for definite statement, but PC neurons were observed in almost equal numbers in areas $3 \mathrm{~b}$ and 1 ; they may be more common in area 1 . The absence of electrode tracks in the brains studied makes laminar designations of neuron locations impossible; no conclusions of this study depend on such designations.

We found the multielectrode recording system of great advantage for studies of the postcentral gyrus, especially when the experiment required that we position microelectrodes in zones within which the glabrous skin of the fingers is represented.

\section{The primate capacity for frequency discrimination in flutter}

Three male human subjects, aged 25, 34, and 71 yr and experienced in psychophysical experiments in flutter-vibration, were tested in the passive discrimination paradigm of Figure 1. Pooled results for the 3, with 50 or more trials per point, are given in Figures 2 and 3. They show the human capacity to make these discriminations and that this capacity did not decline as the testing frequencies were shifted from the range of flutter to that of vibration, at 100 and $200 \mathrm{~Hz}$. The Weber fractions were as follows: at $200 \mathrm{~Hz}, 7 \% ; 100 \mathrm{~Hz}, 11 \% ; 60 \mathrm{~Hz}, 10 \% ; 40 \mathrm{~Hz}, 7 \%$; $30 \mathrm{~Hz}, 7 \%$; and $20 \mathrm{~Hz}, 17 \%$. The stimuli of different frequencies were equalized for subjective magnitude, and our control experiments indicated that our subjects were discriminating between the frequencies and not the intensities of the stimuli they compared.

We then tested 1 human subject (male, aged $34 \mathrm{yr}$ ) in the active mode of the frequency-discrimination task (Fig. 1). His performance is shown in Figure 4. The Weber fractions were as follows: at $30 \mathrm{~Hz}, 4 \% ; 40 \mathrm{~Hz}, 4 \% ; 60 \mathrm{~Hz}, 7 \%$; and $100 \mathrm{~Hz}, 4 \%$. These compare with Weber fractions for this same subject when working in the passive mode of the task: at $30 \mathrm{~Hz}, 5.4 \% ; 40 \mathrm{~Hz}$, $6.5 \%, 60 \mathrm{H}, 7 \%$; and $100 \mathrm{H}, 9.5 \%$. His performance was slightly better in the active than in the passive mode of frequency discrimination. It is well known that an atonal interval exists in the sense of flutter-vibration. It is a range of stimulus amplitudes in which subjects can detect but cannot discriminate 


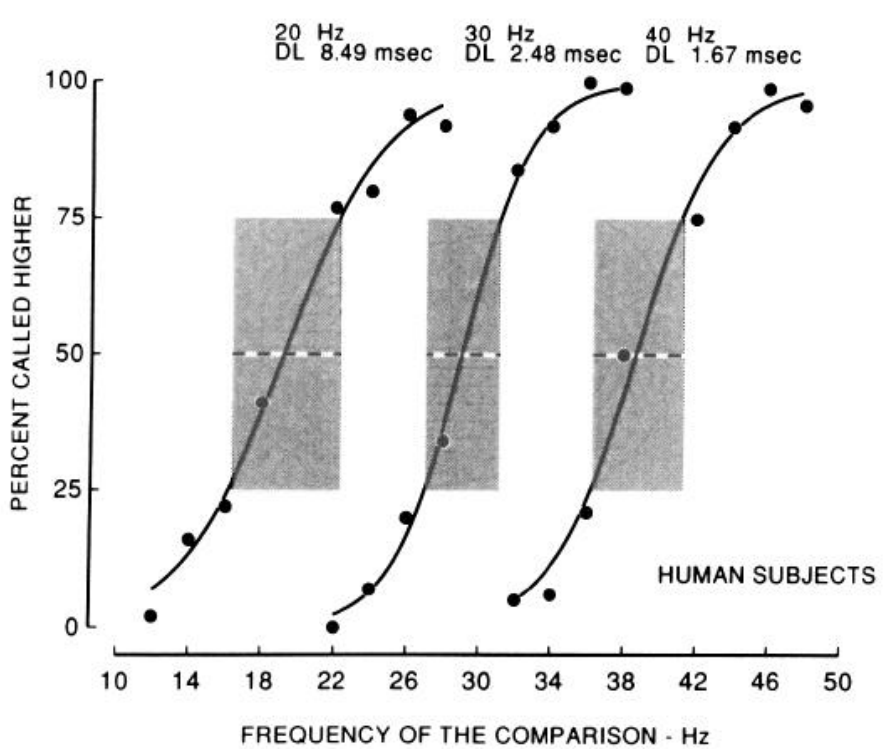

Figure 2. Averaged results for 3 experienced male human subjects, aged 25,34 , and $71 \mathrm{yr}$, working in passive frequency-discrimination task of Figure 1, with base frequencies of 20,30 , and $40 \mathrm{~Hz}$. Data points are means of 60 or 90 trials and indicate the percent trials in which the frequency of the comparison stimulus was identified as higher than that of the base stimulus. The curves are logistic functions fitted to these data points. DL was calculated as described in the text. All stimuli are equated for equal subjective magnitude and delivered at $10 \times$ thresholds.

between frequencies. We have measured this interval in both the active and passive tasks of Figure 1 with the same human subject (male, aged $34 \mathrm{yr}$ ). The results are given in Figure 5. They show a slightly better performance for this subject in the

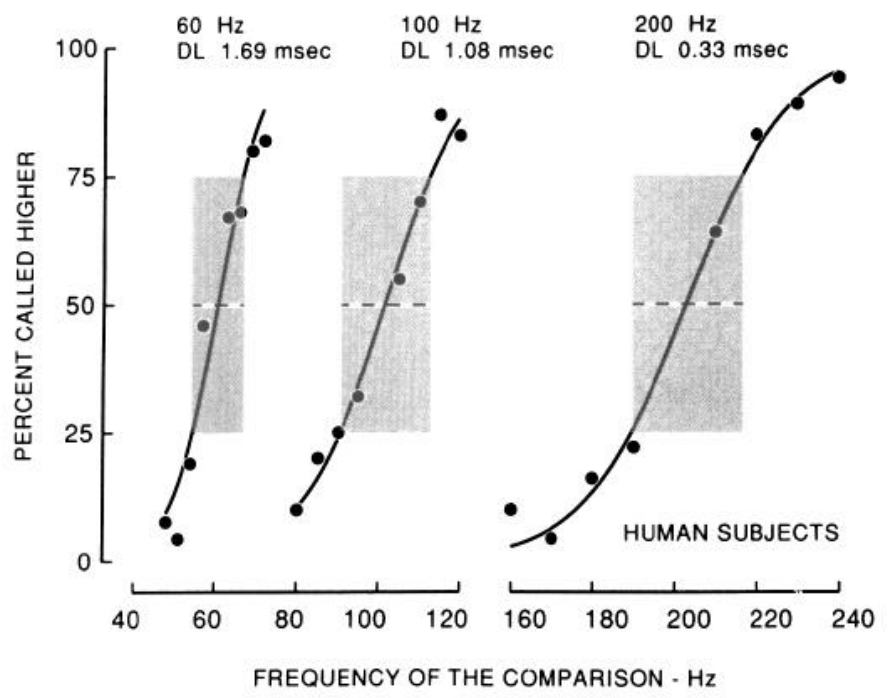

Figure 3. Averaged results for 3 experienced male human subjects, aged 25,34 , and $71 \mathrm{yr}$, working in passive frequency-discrimination task of Figure 1, with base frequencies of 60,100 , and $200 \mathrm{~Hz}$. Data points are means of 60-90 trials each and indicate the percent of trials on which the frequency of the comparison stimulus was identified as higher than that of the base stimuli. The curves are logistic functions fitted to those data points. DL was calculated as described in the text. All stimuli are equated for equal subjective magnitude and delivered at $10 \times$ thresholds. The Weber fraction does not increase with the frequency of the base stimulus over the range of frequencies tested for the data of Figures 2 and $3(20-200 \mathrm{~Hz})$.

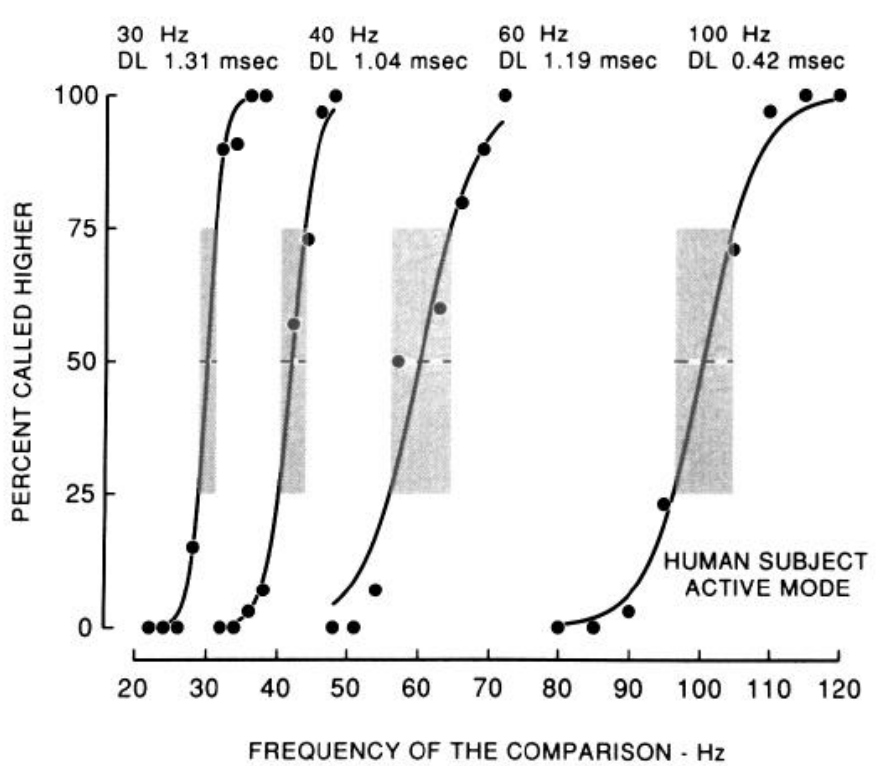

Figure 4. Results obtained in study of 1 human subject (male, aged $34 \mathrm{yr}$ ) working in active frequency-discrimination task outlined in Figure 1 , with base frequencies of $30,40,60$, and $100 \mathrm{~Hz}$. Data points indicate the percent of trials on which the frequency of the comparison stimulus was identified as higher than that of the base stimulus (20-30 trials per point). The curves are logistic functions fitted to those data points. DL was calculated as described in the text. All stimuli were equated for equal subjective magnitude and delivered at $10 \times$ thresholds. Weber fractions are as follows: for $30 \mathrm{~Hz}, 4 \% ; 40 \mathrm{~Hz}, 4 \% ; 60 \mathrm{~Hz}, 7 \%$; and $100 \mathrm{~Hz}, 4 \%$. Force windows were $70-140$ grams for all runs. Discrimination capacity did not decrease with transition of the base stimuli from low- to high-frequency ranges of flutter-vibration. The capacities shown are better than the averages of the subjects of the passive tests of Figure 3, but the 2 performances differed only slightly for this particular subject.

active as compared with the passive mode, but the ranges of the atonal intervals were similar for the 2 modes of stimulus acquisition. It was on the basis of this evidence that we proceeded to combined experiments in monkeys using the passive form of the discrimination task. The amplitude in each stimulus in the frequency-discrimination control sets was set at 10 times the average monkey detection thresholds $(20 \mathrm{~dB})$.

The results obtained for monkey 109 are given in Figure 6. They were obtained after the training period, in a preexperimental phase in which the animal worked with its head fixed. The results show that the monkey exceeded the average performance of our 3 human subjects; his performance, however, was only slightly better than that of our best human subject. Weber fractions for this monkey subject were as follows: at 20 $\mathrm{Hz}, 7 \% ; 30 \mathrm{~Hz}, 6 \%$; and $40 \mathrm{~Hz}, 4 \%$. The results in this monkey are based on the pooled averages of 7 runs at $20 \mathrm{~Hz}$ ( 70 trials/ point), of 12 runs at $30 \mathrm{~Hz}$ (120 trials/point), and of 17 runs at $40 \mathrm{~Hz}$ (170 trials/point). Monkey 108 was trained in a similar manner. His capacities equaled those of our human subjects; his Weber fractions were as follows: at $20 \mathrm{~Hz}, 7 \% ; 30 \mathrm{~Hz}, 7 \%$; and $40 \mathrm{~Hz}, 5 \%$ (all were obtained in single runs during a single day of testing).

\section{The neural discriminandum for frequency}

We describe here the results obtained for 2 postcentral neurons, one studied in the combined experiment in which the stimuli guided behavior and another studied in an alert animal for 

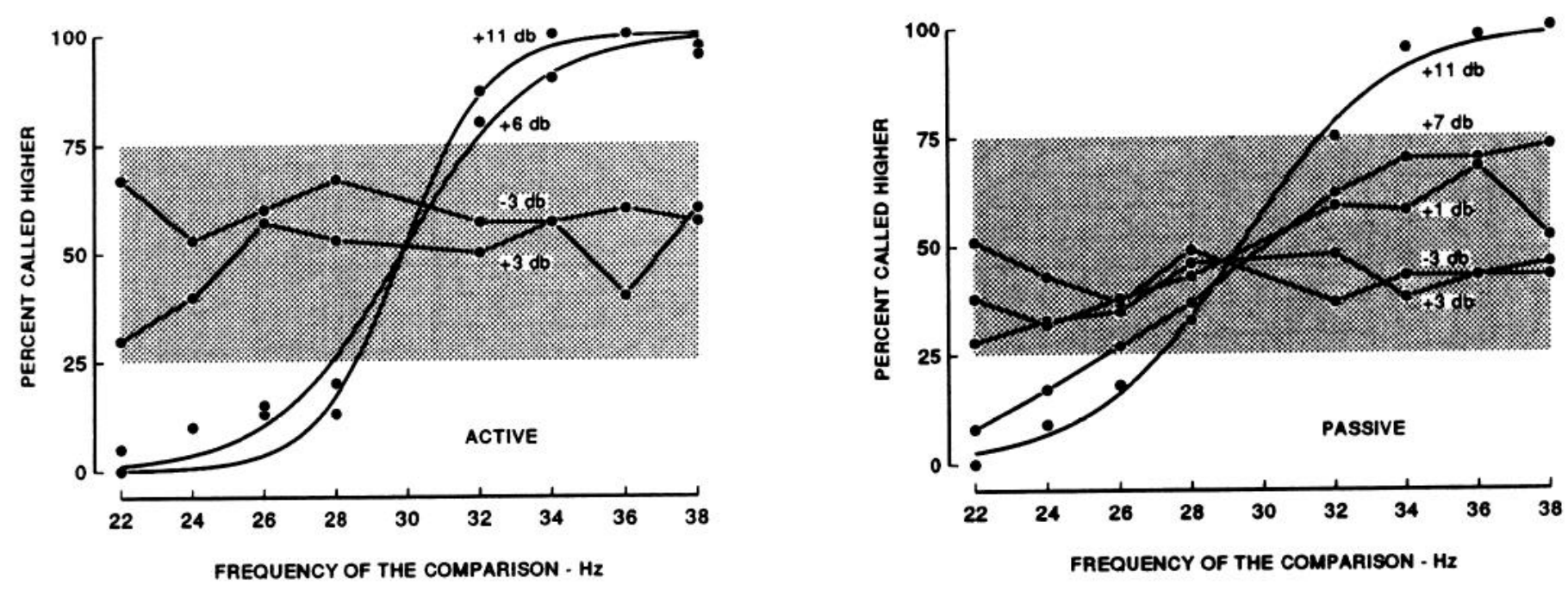

Figure 5. The atonal interval. Results were obtained in a study of 1 human subject (male, aged 34 yr) working in frequency-discrimination tasks, with a base frequency of $30 \mathrm{~Hz}$, at different stimulus amplitudes. Right, Active-task, psychometric functions, at different stimulus amplitudes relative to this subject's threshold at $30 \mathrm{~Hz}(7 \mu \mathrm{m})$, define the atonal interval. Left, Results obtained as the same subject worked in the passive task. The frequency-discrimination limens and the ranges of the atonal intervals differed only slightly in the active and passive modes of stimulus acquisition.

whom the stimuli were irrelevant for behavior, as examples for the data base population. Neuron 700 was isolated $2508 \mu \mathrm{m}$ beneath the top of neural activity in the postcentral gyrus of monkey 109 , in area $3 \mathrm{~b}$. Its receptive field occupied all of the glabrous skin of the distal phalanx of the contralateral third finger. We established the cell as a QA neuron by observing its brief on-off discharges evoked by step indentations of the skin, at different onset slopes. A tuning study revealed that the cell responded best at or near a stimulus frequency of $20 \mathrm{~Hz}$. We then initiated the discrimination task with $20 \mathrm{~Hz}$ as the base frequency. The results are given in Figure 7 . The graph to the right plots the performance of the monkey in this task; the DL for this single run is $4.5 \mathrm{msec}$, which yields a Weber fraction of $9 \%$. The spike replicas to the left of Figure 7 are those of the neural responses evoked in the same run by the base stimuli of $20 \mathrm{~Hz}$, followed by comparison stimuli of $12,16,18,22,24$, and $28 \mathrm{~Hz}$, from above downward, as the animal worked to discriminate between them. Expectation density analysis of these records revealed that the neuron was strongly entrained in a periodic discharge at both the base and comparison frequencies and that the neuronal period lengths matched the relevant stimulus cycle lengths. The frequencies of the neural discharges evoked by base and comparison stimuli did not differ significantly (see Fig. 11).

Figure 8 displays the response patterns of another postcentral QA neuron (634; area 1; depth, $1400 \mu \mathrm{m}$ below the top of neural activity) studied under different circumstances. The stimuli in this case were delivered to the small receptive field on the distal tip of the contralateral second finger of an alert, waking monkey (107), not working in the discrimination task. They were irrelevant for him in the sense that they did not control his behavior. We give evidence below that the behavioral relevance or irrelevance of somesthetic stimuli has no discernible effect upon the postcentral neuronal responses evoked by them. The first 2 columns at the left of Figure 8 are replicas of the neuronal impulse trains evoked by the base and comparison stimuli for selected classes of the control set used. They show, at the extreme left, the onset bursts of activity evoked as the stimulus probe indented the skin and the strong additional activity evoked by the superimposed mechanical sinusoids of the first stimuli, all at $20 \mathrm{~Hz}$, and of the second stimuli, at $12,18,20,22$, and 26 $\mathrm{Hz}$, from above downward. Trained monkeys readily discriminated between any of these stimulus pairs (see Fig. 6). The

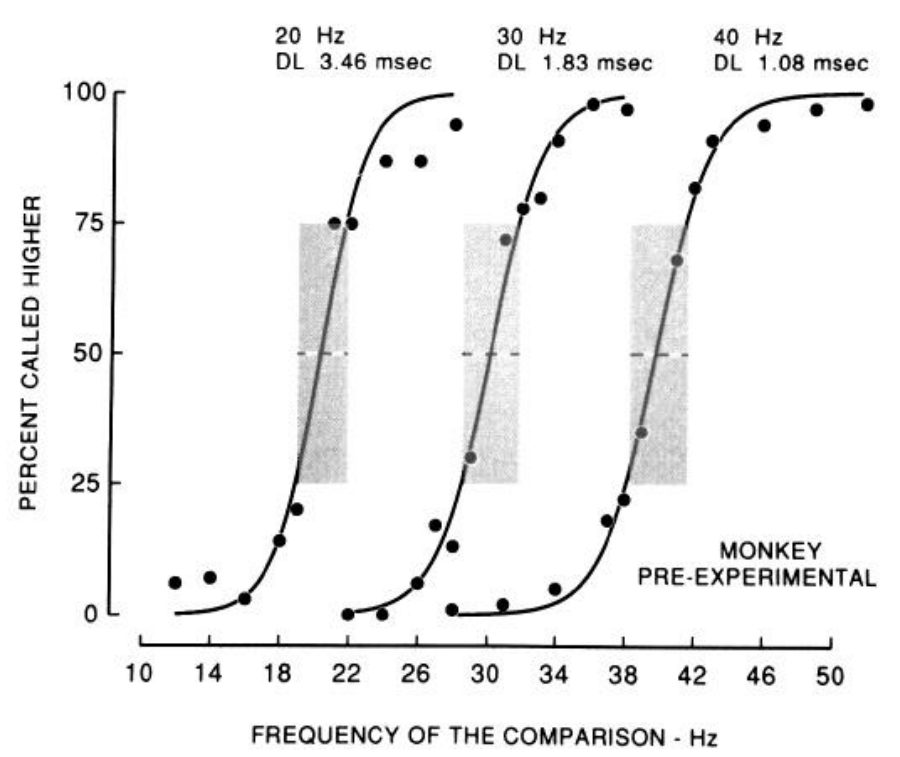

Figure 6. Performance of monkey 109 in passive frequency-discrimination task of Figure 1, with base frequencies of 20,30, and $40 \mathrm{~Hz}$. Psychophysical experiments were made after training to minimal DL in a preexperimental testing period, with the head fixed. Otherwise, circumstances are similar to those of the human task described in the text. Data points show the percent trials on which the frequency of the base stimulus was identified as higher than that of the comparison stimulus (70-170 trials per point). DL was calculated as described in the text. Weber fractions were as follows: at $20 \mathrm{~Hz}, 7 \% ; 30 \mathrm{~Hz}, 7 \% ; 40$ $\mathrm{Hz}, 5 \%-$ not significantly better than those of our most skilled human subject. 



Figure 7. Examples of data obtained in monkey 109 as he worked in combined psychophysical-neurophysiological experiment, in passive discrimination task of Figure 1, with base frequency of $20 \mathrm{~Hz}$. The psychometric function to the right shows his behavioral performance. Data points show percent trials on which the frequency of the comparison stimulus was identified as higher than that of the base stimulus (10 trials per point). The curve is a logistic function fitted to those data points. DL was calculated as described in the text. The Weber fraction is $9 \%$. Spike replicas to the left, obtained simultaneously, are of the discharges of a quickly adapting neuron of the postcentral somatic sensory cortex, evoked by the pairs of stimuli $\left(S_{1}, S_{2}\right)$ the animal received and discriminated between. Each line is the replica of a single trial; each short upstroke is the instant at which an impulse was discharged. Steps indicate stimulus times. Only 6 of the 8 classes of stimuli presented for comparison are shown. Expectation density and harmonic analysis showed periodic entrainment by both the base and comparison stimuli, with period lengths equal to stimulus cycle lengths, as shown for another neuron in Figure 8 . The difference in period lengths is the cortical neural discriminandum in frequency discrimination, at the level of the postcentral gyrus. Neuron 700 is located in area $3 \mathrm{~b}, 2508 \mu \mathrm{m}$ below the top of neural activity, with the receptive field on the distal phalanx of the contralateral third finger.

responses evoked by the base-comparison pairs do not differ significantly in overall frequency. The third and fourth columns of Figure 8 show the expectation density histograms constructed from analyses of the responses replicated in columns 1 and 2 . They reveal the periodic entrainment of the responses of the cortical neuron, and measurement showed that the period lengths in the neuronal patterns are virtually identical to the cycle lengths of the peripheral stimuli evoking them. There are clear differences in the cycle lengths of the neuronal periods in the responses evoked by stimuli between which the monkey can readily discriminate.

These records and analyses are typical of all our studies of postcentral neurons in frequency-discrimination experiments. The periodic neural signal depends on the serial order in which cortical neurons discharge impulses, for the periodicity is almost completely destroyed by a random shuffle of that serial order. This is shown by the renewal density histograms for the responses to comparison stimuli in the fifth column of Figure 8. How little was contributed to periodicity by the presence of impulse intervals at or near the cycle lengths of stimuli is in- dicated by the superimposed impulse interval histograms of the fifth column of Figure 8.

\section{Harmonic analysis of postcentral neuronal responses}

We then sought to measure by Fourier analysis the strength and harmonic content of the periodic signals in the responses of postcentral neurons. The results of such analyses averaged for 17 postcentral QA neurons are shown in Figure 9. These neurons were studied in monkey 109 as he worked in the frequencydiscrimination task, that is, when the delivered stimuli controlled his behavior. A neuron was entered into this population if analysis revealed that any one of the comparison stimuli evoked responses that contained $2 \%$ or more of the total power at the stimulus frequency. Analyses were made in $1-\mathrm{Hz}$ steps over the range from 1 to $500 \mathrm{~Hz}$, but only the results of analyses at the base and comparison frequencies are shown in Figure 9. The power at other test frequencies was consistently less than $0.5 \%$ of the total power, except for the harmonics evoked in some neural responses to low-frequency comparison stimuli. Figure 9 illustrates the powerful, restricted harmonic content of the 
IMPULSE REPLICAS

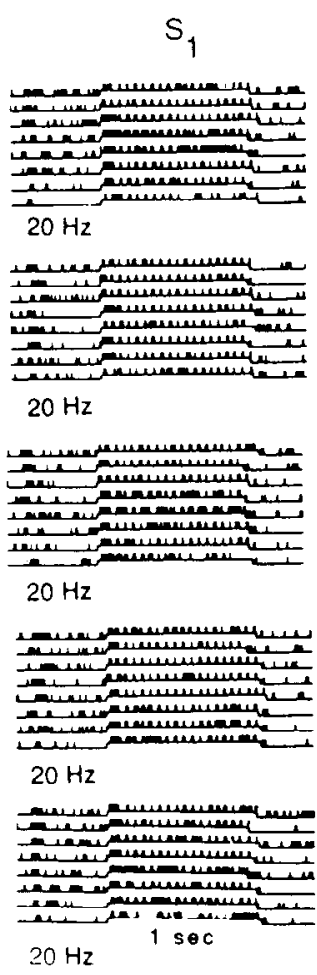

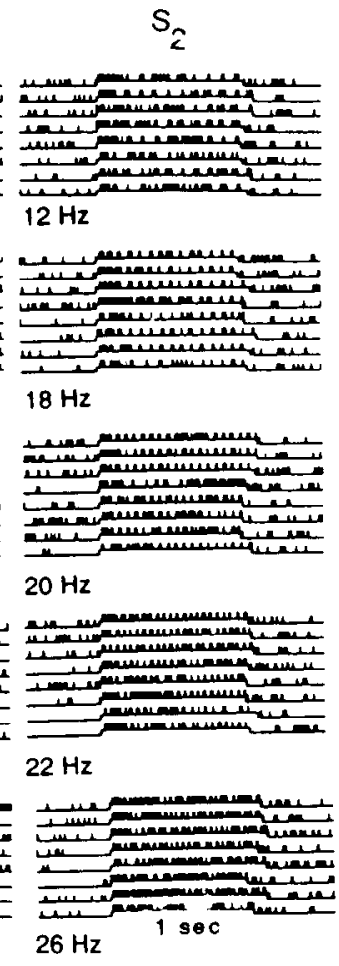

EXPECTATION DENSITY


RENEWAL DENSITY

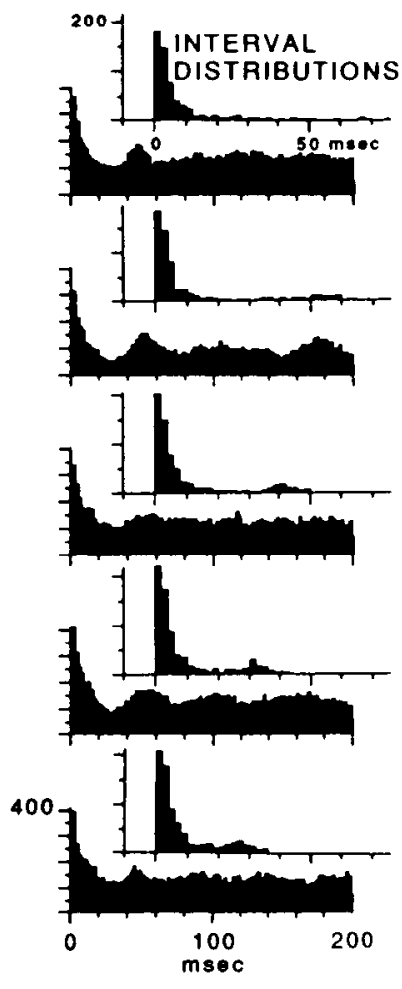

Figure 8. Results obtained in study of postcentral neuron in alert monkey, 107, not working in any task. The stimuli were irrelevant for his behavior. Stimulus sequences were as for the passive task of Figure 1. Columns 1 and 2 , Spike replicas of responses evoked by the base $\left(S_{1}\right)$ and comparison $\left(S_{2}\right)$ stimuli in the frequency discrimination set with base stimulus of $20 \mathrm{~Hz}$; only 5 of the 8 classes presented are shown. Each line is the record of the responses of 1 trial, and each short upstroke is the instant of impulse discharge. Columns 3 and 4 , Expectation density histograms obtained by analysis of the neural records of Columns $I$ and 2. They show the strong periodic entrainment of the neural activity, with period lengths that match exactly the cycle lengths of the stimuli that evoked them, as shown by harmonic analysis. Column 5 , The renewal density histograms for the responses of Column 2 show that the periodic nature of the neural responses depends upon the serial order in which impulses occur, for the periodicity is grcatly reduced by a random shuffle of impulse interval sequences. The inset histograms of impulse interval distributions in Column 5 show that the periodic signal is not due to the presence of large numbers of intervals at or near the stimulus cycle lengths. The differences in the period lengths in the entrained neural activity are regarded as the neural discriminanda at the level of the postcentral somatic sensory cortex for frequency discrimination. Neuron 634 is a QA cell of area $1,1400 \mu \mathrm{m}$ below the top of neural activity; stimuli were delivered to its receptive field on the glabrous skin of the tip of the second finger contralateral to the recording site.

responses evoked in this set of postcentral QA neurons by both the base and comparison stimuli. For example, the set responded to the base stimuli of $20 \mathrm{~Hz}$ with periodic signals containing an harmonic component, at $20 \mathrm{~Hz}$, of $8-10 \%$ of the total power in the signal. Moreover, these same neurons responded, after a 1 -sec delay, with an equally powerful periodic signal in which the harmonic content shifted dramatically to the frequency of the comparison stimulus; for example, compare the sets of 20 versus $22 \mathrm{~Hz}$ or 20 versus $18 \mathrm{~Hz}$ shown in Figure 9. Our analyses at $1-\mathrm{Hz}$ steps revcaled that the signal power at test frequencies only $1-2 \mathrm{~Hz}$ away from stimulus frequencies dropped to background levels. It is clear that the frequencies of the 2 stimuli compared in the frequency-discrimination task are represented in the responses of the postcentral QA population in a clear and powerful way as the lengths of the periodicities in the neural signals evoked by those stimuli; they match the cycle lengths of the evoking stimuli. We conclude that these differences in the neuronal period lengths serve as the critical neural discriminanda underlying the primate capacity to make the frequency discriminations in the range of flutter shown in Figures 2 and 4-6.

\section{Postcentral neuronal response independent of stimulus relevance}

An important question in studies of the central neural processing mechanisms leading to perception is this: At what stage in those processes are the powerful effects of attention and of other central control states exerted? We have examined a number of postcentral QA neurons in our frequency-discrimination paradigm, but with stimuli delivered to an untrained but alert monkey not working in the discrimination task (monkey 107). Harmonic analysis of the results have been made in the manner described above. The results for 25 postcentral QA neurons were averaged and are shown in Figure 10. They replicate almost exactly the results shown in Figure 9 obtained in discriminating monkey 109. Indeed, subtraction of the matrix of Figure 10 from that of Figure 9 left remainders that fell into the range of background. We conclude that whether pairs of stimuli are relevant or irrelevant for guiding sensory-perceptual behavior makes no difference in the responses of QA neurons of areas $3 \mathrm{~b}$ and 1 activated by those stimuli. We emphasize that the definition of relevant and irrelevant states contains no inference concerning 


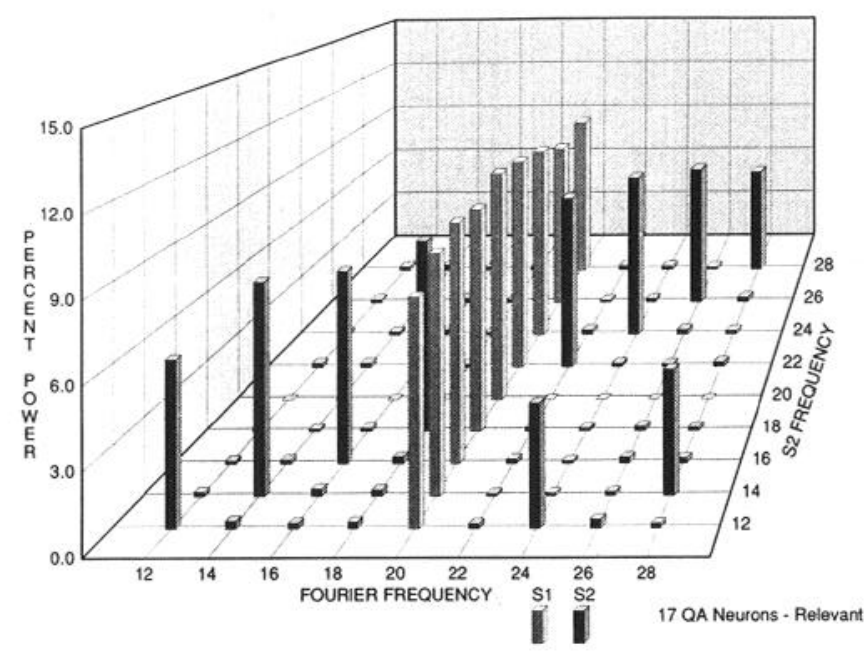

Figure 9. Results of Fourier analysis of responses of 17 QA neurons. These neurons are of areas $3 \mathrm{~b}$ and 1 of the postcentral somatic sensory cortex of monkey 109 as he worked in the passive task of Figure 1, with base frequency of $20 \mathrm{~Hz}$. Methods of analysis are as described in the text. Light columns indicate percent of total spectral power (range of analysis, 1-500 Hz) evoked at the frequency of the base stimulus. Dark columns show the percent total spectral power evoked by the comparison stimuli at their frequencies, from 12 to $28 \mathrm{~Hz}$, in different rows. Power at second harmonics is significant at comparison frequencies of 12 and $14 \mathrm{~Hz}$, but not at others. Therefore, the same set of cortical neurons delivers, with a 1-sec interval, robust signals of the frequencies of the base and comparison stimuli presented for discrimination. The difference between the 2 power spectra is regarded as the neural discriminandum at the level of the postcentral somatic sensory cortex for those frequency discriminations.

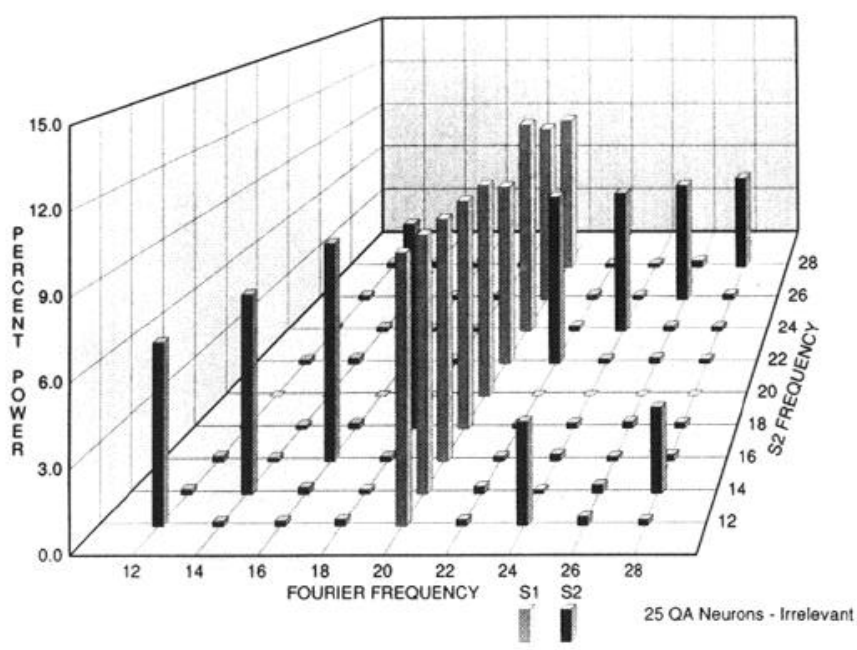

Figure 10. Results of Fourier analysis of responses of 25 QA neurons of areas $3 \mathrm{~b}$ and 1 of postcentral somatic sensory cortex of monkey 107 . Stimuli were delivered in the sequence of the passive task of Figure 1, but the stimuli were irrelevant for the behavior of this monkey, who worked at no task. The results duplicate almost exactly those of Figure 9 , obtained in monkey 109 , as he made discriminations in the passive task, and for whom these same stimuli were relevant for sensory behavior. Subtraction of the matrix of Figure 9 from that of Figure 10 left only insignificant residuals. The responses of postcentral neurons in areas $3 \mathrm{~b}$ and 1 to mechanical sinusoids are not influenced by the relevance or irrelevance of those stimuli in guiding behavior.

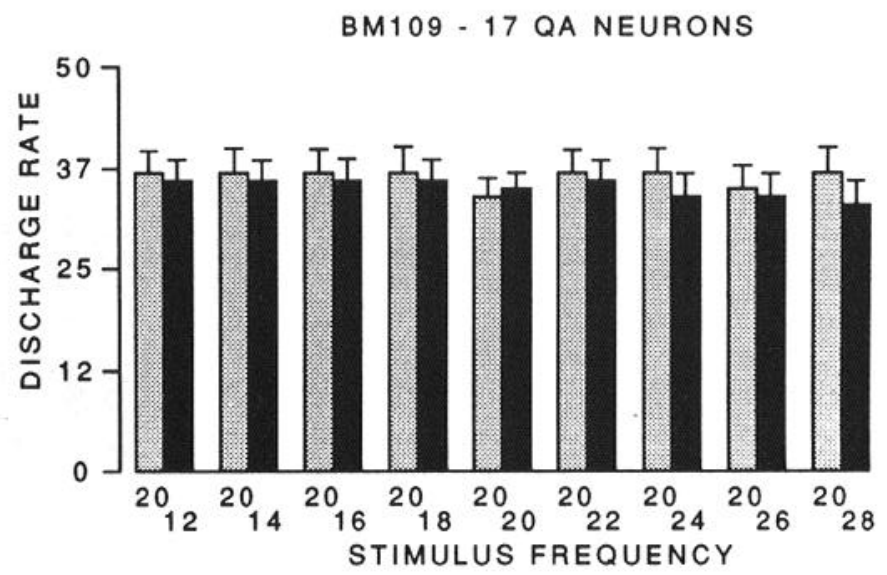

Figure 11. Results of analysis of discharge rates in responses of $17 \mathrm{QA}$ neurons of areas $3 \mathrm{~b}$ and 1 of postcentral sensory cortex of monkey 109 . The results of harmonic analysis are shown in Figure 9. Recordings were made as the monkey performed the passive frequency-discrimination task of Figure 1; the stimuli were relevant for behavior. Discharge rates are expressed as impulses per sec and are the averages of the mean discharges for each neuron, themselves averages of the 5-10 trials in each class. The shaded columns plot the discharge rate in the responses to the base stimuli ( $20 \mathrm{~Hz}$ for all classes). The solid columns plot discharge rates in the responses to the comparison stimuli, which differ in all except the 20/20 class. Vertical lines indicate 1 SEM. Responses to comparison stimuli were consistently slightly lower in frequency than those to the base stimuli, which we attribute to a mild forward masking effect. The capacity of monkeys to discriminate between stimuli cannot depend on the frequency of discharge; a neural rate code will not suffice.

where attention is allocated in the 2 states, though all our human subjects reported that the frequency-discrimination task required intense attention to each of the pairs of stimuli.

\section{Neuronal rate code and frequency discrimination}

The responses of the $17 \mathrm{QA}$ neurons analyzed by harmonic analysis for Figure 9 were also analyzed from the standpoint of frequency of discharge without reference to the serial order of neuronal-impulse discharge. Averages were calculated from the responses of each neuron over the $1-\mathrm{sec}$ period of all trials. Population means and their standard errors are shown in Figure 11. The overall frequency of discharge evoked by the base and comparison stimuli for the population of QA neurons considered were virtually identical. We conclude that frequency discriminations cannot be made on the basis of differences in the frequencies of discharge evoked by the stimuli discriminated. The frequencies of the responses to the comparison stimuli are consistently (but not significantly) lower than those of the responses to the base stimuli. We attribute the difference to a weak feed-forward masking effect; we have not examined its time course.

\section{Postcentral signal provided by the SA system}

Figure 12 displays the result of harmonic analysis averaged for a group of SA neurons activated in the frequency-discrimination paradigm in a monkey (107) for whom the stimuli were behaviorally irrelevant. Comparison of these results with those for a set of QA neurons studied under the same conditions in the same monkey (Fig. 10) is sufficient to conclude that the SA system provides sets of strongly periodic signals at the postcentral level, evoked by mechanical sinusoids between which discrimination can be made. Moreover, the frequency ranges of 


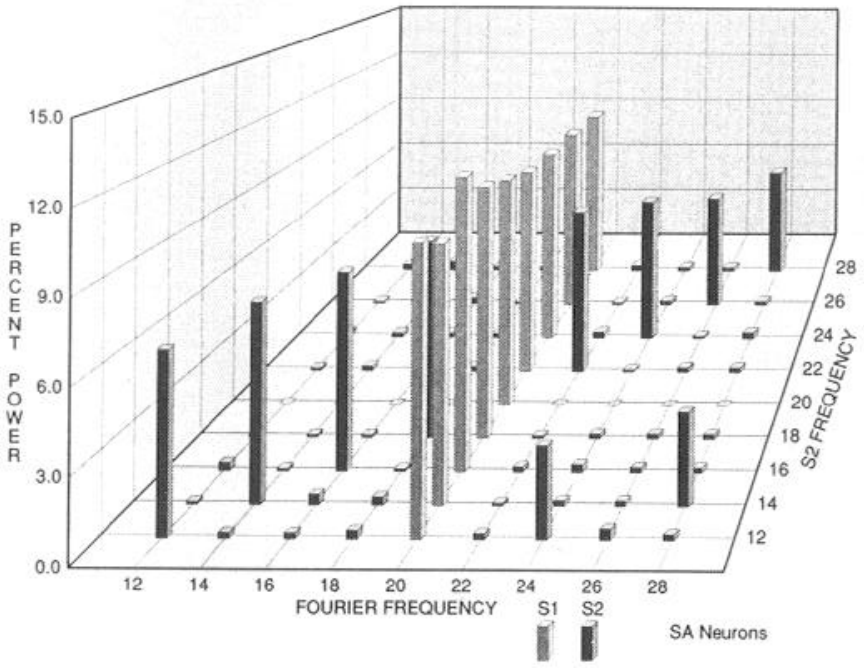

Figure 12. Results of Fourier analysis of responses of set of 9 SA neurons of areas $3 \mathrm{~b}$ and 1 of postcentral somatic sensory cortex. Recordings were made in monkey 107 as he received the stimulus sequence $\left(S_{1}, S_{2}\right)$ of the passive task of Figure 1. This alert animal did not work at the task; for him, the stimuli were irrelevant and did not guide behavior. The results obtained almost exactly duplicate those of QA neurons of the postcentral gyrus shown in Figures 9 and 10. Discharge frequency analysis for this set (not shown) was similar to that illustrated in Figure 11 for QA neurons. Reasons are given in the text for concluding that the SA neurons do not contribute to frequency discrimination, even though their band pass and periodic entrainment resemble closely those of the QA population.

sensitivity of the QA and SA systems are virtually identical. We discuss below whether the cortical signals provided by the SA system are used for frequency identification and discrimination.

\section{Discussion}

\section{Primate capacity for frequency discrimination in the range of flutter}

Our psychophysical studies of frequency discrimination confirm and extend earlier studies made in humans and monkeys with different apparatuses and different experimental paradigms (Mountcastle et al., 1969; LaMotte and Mountcastle, 1975). Each primate can make precise frequency discriminations with DLs that yield Weber fractions below $10 \%$. Monkey and man have equal discriminatory capacities, though only our most skilled human subject approached the performance of our 2 young but mature, trained, male monkey subjects. We observed no significant decline in discriminatory performance as the base stimuli changed from the low- to the high-frequency range of the flutter-vibration spectrum. Goff (1967) observed such a decline in her studies, made with a different experimental paradigm.

It has been known for a long time that the perception of the amplitude of a vibratory stimulus changes with increases in stimulus frequency, as both Goff (1967) and von Bekesy (1962) emphasized. We constructed discrimination control sets with this in mind, delivering pairs of stimuli at different frequencies but of equal subjective magnitude. The 2 control experiments described above indicate that we did achieve this equality. It is unlikely, in view of those results, that stimulus pairs such as 20 and $22 \mathrm{~Hz}$, between which primates discriminate with certainty, could be based on intensity differences that have escaped our scrutiny.

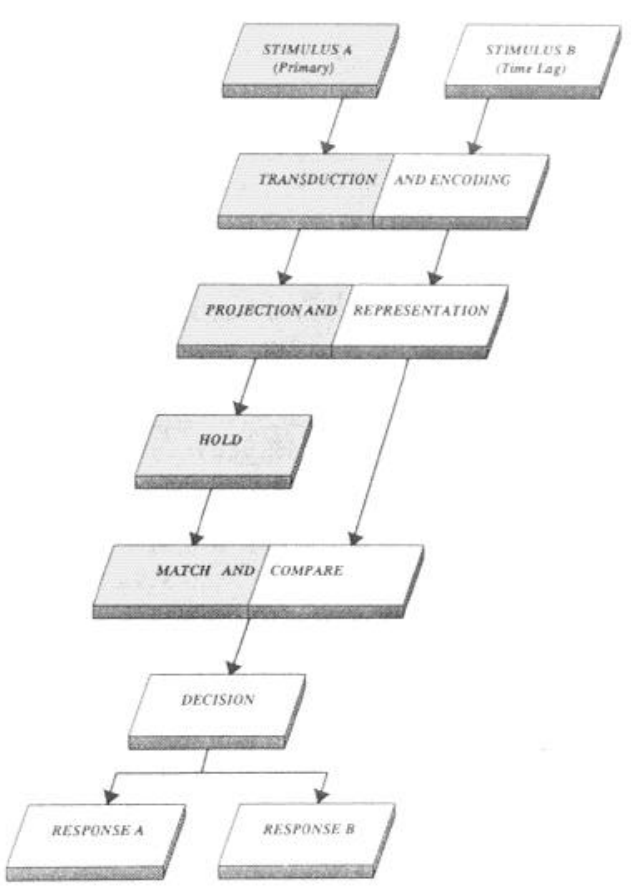

Figure 13. Schematic outline of stimulus-response processes. These processes are thought to lead from the initial transduction and encoding of the periods of mechanical sinusoid stimuli delivered to the hand to the final differentiated motor response that indicates frequency discrimination, indicated by responses $A$ or $B$ (see Discussion).

\section{Postcentral neuronal discriminandum for frequency}

Our general proposition is that frequency discrimination in the range of flutter depends upon a periodic neural code evoked in postcentral neurons by afferent input in the QA system. We define a neural code as a set of neural signals reflecting the physical characteristics of peripheral stimuli and which can be shown to be used for perception and behavioral response. We conclude that the periodic code is adequate for frequency discrimination for the reasons discussed below.

Arguments of suitability and availability. The periodically entrained activity evoked in postcentral QA neurons provides exact signals of the cycle lengths of mechanical sinusoids delivered to the glabrous skin of the hand. The periodic signals evoked by stimuli between which primates can discriminate are sharply and differentially defined, as shown by harmonic analysis (Figs. $9,10)$. These periodic signals are presented in areas $3 \mathrm{~b}$ and 1 of the postcentral somatic cortex. Monkeys cannot make frequency discriminations in the range of flutter after the removal of this cortex (LaMotte and Mountcastle, 1979). Moreover, an earlier study of frequency discrimination in humans made in the context of decision theory revealed a covariation between the degree of uncertainty in frequency discriminations and the variances of the "presumed sensory events," postulated to be the observers' estimates of the period lengths in the periodically entrained activity of postcentral QA neurons (Mountcastle et al., 1969).

Arguments by exclusion. Our data show that frequency discrimination in flutter cannot be based upon a neural rate code, that is, upon differences in the overall rates of discharge evoked in cortical QA neurons by the 2 stimuli discriminated, independent of the internal structure of the evoked neural patterns 
(see Fig. 11). It appears equally unlikely that frequency discriminations in flutter can be based on differences in the spatial distribution within the cortex of the sets of activity evoked by the 2 stimuli discriminated, that is, on a spatial code. The neural discharge evoked by the 2 successive stimuli discriminated occur successively in time in the same sets of first-order afferents and in the same sets of neurons of the dorsal column nuclear complex, thalamic ventrobasal nuclear complex, and postcentral somatic sensory cortex. A place theory like that successful in understanding frequency discrimination in audition is also inappropriate, for the best frequencies and the frequency bandwidths of elements of the QA system from skin to cortex are virtually identical to one another. Finally, it appears doubtful that the frequency discriminations we observed were based on different degrees of differential activation of the QA and PC systems, because (1) it is unlikely that stimuli so close in frequency (e.g., 20-22 Hz) would evoke such different differentials, (2) elegant frequency discriminations can be made in primates at frequencies and amplitudes of stimuli that are unlikely to engage the $\mathrm{PC}$ system at all, and (3) frequency discriminations in the PC frequency range can be made after local anesthesia of the glabrous skin has eliminated the QA and other cutaneous afferent systems [M. M. Merzenich and T. Harrington, unpublished observations (1969)].

\section{Relevance or irrelevance of stimuli and direction of attention}

The powerful effect of the allocation of attention in determining the outcome in sensory detection and discrimination tasks is well known from many studies in sensory psychophysics (for reviews, see Posner, 1978; Kahneman and Treisman, 1984). The neural mechanisms of attention and how it affects sensory processes in the brain are unknown, but some inferences concerning them may be derived by defining the locations in the central neural processing networks at which that effect is imposed. The majority of studies made in humans with the eventrelated evoked-potential method suggest that attention effects are imposed at a point central to the initial representation of sensory events in primary sensory cortical areas. Our observation that the relevance or irrelevance of stimuli does not affect the responses of neurons in areas $3 b$ and 1 is consonant with that view, for the physical features of those stimuli are represented robustly in the activity of postcentral neurons, whether or not those stimuli guide behavior. However, there is uncertainty concerning where attention is allocated during the irrelevant-stimulus condition. What is certain, as reported by our human subjects, is that concentrated attention to the stimuli is required for success in our frequency-discrimination tasks, and the slightest disturbance (e.g., aberrant sounds) caused transient deteriorations in the performances of our monkeys in the discrimination task.

Desmedt and Tomberg (1989) have shown in their studies of the event-related potentials recorded from the scalp over the postcentral gyrus in human subjects, evoked by electrical stimulation of the contralateral fingers, that only the very earliest components, the $\mathrm{P} 10 / \mathrm{N} 20$ complex, are completely exogenous and immune to change by the allocation of attention to or away from the evoking stimuli. The quickly following P27 component, assigned by these authors to the generation of a dipole in area 1 , can be affected by such allocation. Thus, both monkey and human studies converge to the conclusion that the neural responses in area $3 \mathrm{~b}$ are unaffected by stimulus relevance and that the effect of attention is imposed at a later stage; whether this stage is in area 1, as the human studies suggest, or in area 2 or 5 , as we infer from our own results, remains to be determined.

\section{Active versus passive modes of stimulus acquisition}

The mode of stimulus acquisition, whether active or passive, does not affect the somesthetic sensory performance of the primate. For example, the capacities for discrimination between surfaces with different textures (Lamb, 1983), between surfaces of different degrees of roughness (Lederman, 1983), or in identifying embossed letters of the alphabet (Grunwald, 1978) are the same, independent of whether the subject actively scans his hand across the surfaces or whether the surfaces are moved across the skin of the immobile hand, at the same speeds. Moreover, performances were only $25 \%$ better when subjects scanned stationary letters compared to static palpation alone (Phillips et al., 1983). Scanned touch appears to be only slightly if at all better than stationary touch (Phillips and Johnson, 1985). Our comparison between active and passive modes differs from those described, for in neither mode is the receptive surface moving. The active mode we have used is a combination of 2 tasks. The subject must first maintain pressure on the manipulandum within a set range, monitoring that pressure by visual inspection of indicator lights, and then discriminate between the frequencies of 2 superimposed mechanical sinusoids. Only the latter is required in the passive task. The results suggest that the motor actions required in the active task do not generate a significant central reentrant facilitatory or inhibitory effect on the neural operations of detection or discrimination in the sense of flutter.

\section{Postcentral neuronal responses to flutter stimuli and atonal interval}

Observations in humans and monkeys have shown consistently that frequency discriminations cannot be made in the frequency range of flutter until stimulus amplitudes are increased by $6-8$ $\mathrm{dB}$ above detection thresholds. We have confirmed these findings in our current studies and have found also that the atonal intervals differ only slightly for a human working in the active $(6 \mathrm{~dB})$ or the passive $(7 \mathrm{~dB})$ modes of the tasks of Figure 1 (see Fig. 5). Studies of first-order QA fibers innervating the glabrous skin of the monkey hand revealed similarly dual thresholds. The first is that stimulus amplitude just sufficient to evoke any response. The second is that level, some 6-8 dB higher, at which stimuli evoke perfectly entrained neural discharges, 1 impulse per stimulus cycle. For many QA fibers, further increases in stimulus amplitude above this tuning threshold produce no further changes in neuronal discharge until high amplitudes are reached; this is the tuning plateau (Talbot et al., 1968; Mountcastle et al., 1972). This led the latter authors to match these pairs of peripheral and behavioral thresholds. Our studies of the responses of postcentral neurons to stimuli varying over a wide, intensive continuum show no such duality. The weakest effective stimulus evokes postcentral neuronal activity entrained to the stimulus frequency. Further increases in stimulus amplitude evoke a smooth increase in the intensity of the periodically entrained discharge and in the strength of its harmonic signal at stimulus frequency. There is no tuning threshold and no tuning platcau. Such a result is predictable if first-order QA fibers with different tuning points and plateaus converge across the linking neurons of the somatic system onto single postcentral cells, as they do. The atonal interval is a zone of increasing stimulus amplitudes through which the primate capacity for 
frequency discrimination increases gradually towards an arbitrary criterion level. There is no sharp threshold along the amplitude continuum between those stimuli that can be discriminated and those that cannot (see Fig. 5).

\section{Is the SA ststem sufficient for frequency discrimination in the frequencly range of flutter?}

We found that both the SA and the QA sets of postcentral neurons are entrained periodically, via their linking systems to the first-order afferents, by mechanical sinusoids in the frequency range of flutter delivered to the glabrous skin of the primate hand. The strengths of the periodic postcentral neuronal signals and the narrow restriction of harmonic power to stimulus frequencies are identical in the 2 systems. The frequency bandwidths of the 2 systems are identical. This suggests that either the QA or the SA system could provide cortical neuronal signals adequate for frequency identification and discrimination in the frequency range of flutter.

Several other lines of evidence bear upon this question. The first is the interaction between the depth of skin indentation by a stimulus probe, the frequency of the quasi-steady discharge produced in the first-order $\mathrm{SA}$ afferents by that indentation, and the ease with which superimposed sinusoids may evoke a periodic entrainment of that discharge. It has been shown, for the glabrous skin of the monkey's hand (see Figs. 17-19 in Talbot ct al.. 1968), for the skin of the back of the human hand (Konictzny and Hensel, 1977), and for the hairy skin of the monkey's arm (Merzenich and Harrington. 1969), that first-order SA afferents of these different types may be entrained at amplitudes of mechanical sinusoids even an order of magnitude lower than the primate detection thresholds in the frequency range of flutter. Observations in microneuronography experiments in humans also bear upon this question (Torebjork and Ochoa, 1980; Ochoa and Torebjork, 1983; Vallbo et al., 1983, 1984; Torebjork et al. 1984, 1987). Electrical stimulation of single, isolated QA fibers innervating the human glabrous skin evokes a sense of flutter. Similar electrical stimulation of an isolated SA fiber evokes a sense of skin pressure. Change in the frequency of stimulation of the QA fiber produces a change in perceived frequency, whereas change in the frequency of stimulation of the SA fiber produces a change in the perceived intensity of skin pressure.

These facts indicate that the periodic signals in the SA system are not used for frequency discrimination or identification. The implications of such a finding are several. First, what appears to be a neural signal of a sensory event, even at the cortical level. cannot be regarded as a neural code for that event unless it can be shown that the signal is used at the level of perception and behavioral response. Second, it implies that the SA and QA systems are, to a remarkable degree, kept separate from the initial postcentral representations to and through the neural levels of perceptual operations. Third. it implies that the perceptual operations themselves must differ for the 2 systems. Those for the SA system must include an integrative operation that smooths the input and leads to the perception of skin pressure and its variations in intensity. Those for the QA system must specifically be free of such an integrative stage, and variations in the neuronal period lengths of different stimulus frequencies must lead to the perception of differences in the cycle lengths of different stimulus frequencies. Further evidence concerning these perceptual operations awaits a method for selective activation of the $\mathrm{SA}$ and $\mathrm{QA}$ systems at the periphery that will allow independent requirements of the behavior and central neural response of each system.

\section{Some conjectures on the higher-order neuronal mechanisms in} frequency discriminations

Figure 13 illustrates a simple flow diagram of the processes we suspect play a role in frequency discrimination in the sense of flutter. I he evidence presented in this paper indicates that the frequencies of the 2 stimuli discriminated are precisely signaled (represented) by the lengths of the periods in the periodically entrained sets of cortical neuronal activity evoked by the 2 stimuli. It is important to emphasize that the 2 sets of neuronal activities occur sequentially in time in the same set of cortical neurons. Therefore, these activities must be compared sequentially in time. It seems likely that discrimination cannot be made on the basis of differences in the neural populations activated by the 2 stimuli. The neural discriminandum for frequency is the difference in the period lengths in the 2 sets of neural activity.

How are those 2 sets compared? One alternative is that the period of the first set is held in a short term (sensory) memory and compared directly with the period in the second set of activity arriving in the same neural elements as the first. A second alternative is that the period of the first set is identified and used to access a standard template in/trom memory for comparison with the period in the second set of neural activity. Either alternative requires close attention to the frequency of the first stimulus, and all our subjects report that success in the flutter-discrimination task requires intense attention to the frequencies of the 2 stimuli compared. Both monkeys and human subjects have succeeded in making flutter discriminations when working with mixed stimulus control sets that contain 2 or more different base stimuli and their associated comparison stimuli. Under these conditions, base stimuli of different frequencies appear in random sequence, which suggests that each must be identified individually for successful discriminations. whichever of the 2 alternatives is used.

We have not yet observed any signs of the "hold," "compare." or "decision" processes indicated in Figure 13 in the activity of any neurons of areas $3 \mathrm{~b}$ or 1 of the somatic sensory cortex in animals working successfully in the frequency-discrimination task. We interpret this to mean that these higher-order operations-assuming that they are necessary for frequency discriminations-must occur in more central nodes of the distributed somatic sensory networks, particularly in the homotypical cortex of the parietal lobe.

\section{References}

Bloomfield P (1976) Fourier analysis of time series - an introduction. New York: Wiley.

Bracewell RN (1986) The Fourier transform and its applications. New York: McGraw-Hill

Darian-Smith I, Goodwin A. Sugitani M. Heywood J (1984) The tangible features of textured surfaces: their representation in the monkey's somatosensory cortex. In: Dynamic aspects of neocortical function (Edelman GM, Gall WE, Cowan WM, eds), pp 475-500. New York: Wiley.

Desmedt JE, Tomberg C (1989) Mapping early somatosensory evoked potentials in selective attention: critical evaluation of the control conditions used for titrating by difference the cognitive P30, P40, PI00, and N140. Electroencephalogr Clin Neurophysiol 74:321-346.

Draper NR, Smith H (1981) Applied regression analysis, p 709. New York: Wiley.

Fisher RA (1929) Test of significance in harmonic analysis. Proc $R$ Soc Lond [A] 125:54-59.

Gerstein GL (1987) Information flow and state in cortical neural net- 
works: interpreting multi-neuron experiments. In: Organization of neural networks: structures and models (von Steelen W, Shaw G, Leinhos UM, eds), pp 53-75.

Goff GD (1967) Differential discrimination of frequency of cutaneous mechanical vibration. J Exp Psychol 74:294-299.

Grunwald AP (1978) On braille and braille machines. Sensory World $3: 4$.

Iwamura Y, Tanaka M, Hikosaka O (1980) Overlapping representation of fingers in the somatosensory cortex (area 2) of the conscious monkey. Brain Res 197:516-520.

Kaas JH, Nelson J, Sur M, Lin CS, Merzenich MM (1979) Multiple representations of the body within the primary somatosensory cortex of primates. Science 204:521-523.

Kahneman D, Treisman A (1984) Changing views of attention and automaticity. In: Varieties of attention (Parasuraman R, Davies DR, eds), pp 29-62. London: Academic.

Klemm WR, Sherry CJ (1981) Serial ordering in spike trains: what's it "trying to tell us"? Intern J Neurosci 14:15-33.

Klemm WR, Sherry CJ (1982) Do neurons process information by relative intervals in spike trains? Neurosci Biobehav Rev 6:429-437.

Konietzny F, Hensel H (1977) Response of rapidly and slowly adapting mechanoreceptors and vibratory sensitivity in human hairy skin. Pflugers Arch 368:39-44.

Lamb GD (1983) Tactile discrimination of textured surfaces: psychophysical performance measurements in humans. J Physiol 338:551565.

LaMotte RH, Mountcastle VB (1975) The capacities of humans and monkeys to discriminate between vibratory stimuli of different frequency and amplitude: a correlation between neural events and psychophysical measurements. J Neurophysiol 38:539-559.

LaMotte RH, Mountcastle VB (1979) Disorders in somesthesis following lesions of parietal lobe. J Neurophysiol 42:400-419.

Lederman SJ (1983) Tactual roughness perception: spatial and temporal determinants. Can J Psychol 4:498-511.

Merzenich MM, Harrington T (1969) The sense of flutter-vibration evoked by stimulation of the hairy skin of primates: comparison of human sensory capacity with the responses of mechanoreceptive afferents innervating the hairy skin of monkeys. Exp Brain Res 9:236260.

Moore GP, Perkel DH, Segundo JP (1966) Statistical analysis and functional interpretation of neuronal spike data. Ann Rev Physiol 28: 493-522.

Mountcastle VB, Talbot WH, Sakata H, Hyvarinen J (1969) Cortical neuronal mechanisms in flutter vibration studied in unanesthetized monkeys. J Neurophysiol 32:453-484.

Mountcastle VB, LaMotte RH, Carli G (1972) Detection thresholds for stimuli in humans and monkeys: comparison with threshold events in mechanoreceptive afferent nerve fibers innervating the monkey hand. J Neurophysiol 35:122-135.
Ochoa J, Torebjork E (1983) Sensations evoked by intraneural microstimulation of single mechanoreceptive units innervating the human hand. J Physiol (Lond) 342:633-654.

Perkel DH (1970) Spike trains as carriers of information. In: The neurosciences, second study program (Schmitt FO, ed), pp 589-596. New York: Rockefeller UP.

Perkel DH, Gerstein GL, Moore GP (1967) Neuronal spike trains and stochastic point processes. I. The single spike train. Biophys J 7:391418 .

Phillips JR, Johnson KO (1985) Neural mechanisms of scanned and stationary touch. J Acoust Soc Am 77:220-224.

Phillips JR, Johnson KO, Brownc HM (1983) A comparison of visual and two modes of tactual letter recognition. Percept Psychophys 34: 243-249.

Poggio GF, Viernstein LJ (1964) Time series analysis of impulse sequences of thalamic somatic sensory neurons. J Neurophysiol 27: $517-545$.

Posner MI (1978) Chronometric explorations of mind. Hillsdale, NJ: Erlbaum.

Reitboeck HJ (1983a) Fiber microelectrodes for electrophysiological recordings. J Neurosci Meth 8:249-262.

Reitboeck HJ (1983b) A 19-channel matrix drive with individually controllable fiber microelectrodes for neurophysiological applications. IEEE Trans Syst Man Cybern 13:676-683.

Shimshoni M (1971) On Fisher's test of significance in harmonic analysis. Gcophys J Roy Astr Soc 23:373-377.

Talbot WH, Darian-Smith I, Kornhuber HH, Mountcastle VB (1968) The sense of flutter vibration: comparison of the human capacity with response patterns of mechanoreceptive afferents from the monkey's hand. J Neurophysiol 31:302-334.

Torebjork HE, Ochoa JL (1980) Specific sensations evoked by activity in single identified sensory units in man. Acta Physiol Scan 110:445447.

Torebjork HE, Schady W, Ochoa J (1984) Sensory correlates of somatic afferent fibre activation. Hum Neurobiol 3:15-20.

Torebjork HE, Vallbo AB, Ochoa JL (1987) Intraneural microstimulation in man: its relation to specificity of tactile sensations. Brain 110: 1509-1529.

Vallbo AB, Johannson RS (1984) Properties of cutaneous mechanoreceptors in the human hand related to touch sensation. Hum Neurobiol 3:3-14.

Vallbo AB, Olsson KA, Westberg KG, Clark FJ (1984) Microstimulation of single tactile afferents from the human hand: sensory attributes related to unit type and properties of receptive fields. Brain 107: 727-749.

von Bekesy G (1962) Can we feel the nervous discharges of the end organs during vibratory stimulation of the skin? J Acoust Soc Am $34: 850-856$ 